\title{
A case of primary granulomatous hypopysitis that was initially treated as a prolactinoma
}

Arslan E. $.^{1}, \underline{\text { Cakir N. }}{ }^{1}$ Altınova Eroglu A. ${ }^{1}$, Yalçin M.M. ${ }^{1}$, Akyuz P2 ${ }^{2}$, Akturk M. ${ }^{1}$, Balos Toruner F. ${ }^{1}$, Arslan M. ${ }^{1}$

1- Department of Endocrinology and Metabolism, Gazi University, Faculty of Medicine, Ankara, Turkey

2- Department of Internal Medicine, Gazi University, Faculty of Medicine, Ankara, Turkey

\section{Introduction}

Hypophysitis is an inflammatory disease of the pituitary gland. It may either be primary or secondary associated with systemic diseases. Primary hypophysitis is divided into 5 subgroups histologically; lymphocytic, granulomatous, xanthomatous, xanthogranulomatous and necrotizing. Among these, lymphocytic hypophysitis is the most common type. The second most common type is granulomatous hypophysitis.

The most common presenting symptom is headache. Major accompanying symptoms are visual changes, fatigue, polyuria, polydipsia, galactorrhea and erectile dysfunction. Radiologically, diffuse expansion in pituitary gland, extension to suprasellar zone, thickening in infundibulum and compression of the optic chiasm can be observed in patients with hypophysitis. Primary hypophysitis can imitate pituitary adenomas in laboratory and clinical conditions. Diagnosis is usually made histologically following hypophysectomy or pituitary biopsy.

In this study, we reported a case that was initially misdiagnosed and treated as prolactinoma but then definitively diagnosed as granulomatous hypophysitis as the result of transsphenoidal pituitary biopsy.

\section{Case Report}

A 50-year-old female patient presented to an Obstetrics and Gynecology Hospital with headache and menstrual irregularities over the preceding year. On the visual field examination made by Ophthalmology Department, visual defect (close to complete total defect on right eye) and a peripheral visual field defect on left eye's lower nasal and temporal area have been detected. The pituitary MRI demonstrated a macroadenoma around $17 \times 9 \times 12 \mathrm{~mm}$, substantially filling the pituitary gland, extending to optic chiasm and filling the suprasellar cistern (Figure 1). In laboratory tests, TSH was $1,99 \mathrm{ulU} / \mathrm{L}(0,35-4,97)$ and prolactin level was detected as $115 \mathrm{ng} / \mathrm{ml}(1,9-25)$. Cabergoline therapy was administered to the patient.

When she presented to our Clinic, no significant pathology was found on physical examination. Bilateral peripheral visual field defect was detected in visual field. The laboratory tests values were: IGF-1: $106.6 \mathrm{ng} / \mathrm{ml}(101-135), \mathrm{GH}: 0.72 \mathrm{ng} / \mathrm{ml}(0-$ 8), ACTH (morning): $16.7 \mathrm{pg} / \mathrm{ml}(0-46)$, cortisol (morning): 0.882 ug/dl (6.2-19.4), PRL: 1,06 ng/dl, Tsh: 2.9 ulU (0.2-4.2), sT3: $1.41 \mathrm{pg} / \mathrm{ml}(2-4.4), \mathrm{sT} 4: 0.46 \mathrm{ng} / \mathrm{dl}(0.9-1.7)$. The cabergoline therapy was stopped due to the patients' history and prolactin level of 1,06. Upon detection of secondary hypothyroidism and low cortisol levels, hydrocortisone and then levothyroxine therapies were started. In the follow-up pituitary MRI screenings, pituitary gland was measured as $13 \times 11 \times 22 \mathrm{~mm}$, pituitary gland was contrasted in heterogeneous character, and nodule formation with a diameter of $6 \mathrm{~mm}$ was detected in pituitary gland right hemisphere (microadenoma?) (Figure 2).
We were aware of the possibility that this lesion could have been an adenoma responding to cabergoline therapy. But the reexamination of initial and follow-up MRI screenings revealed infundibular thickness and an increase in pituitary gland size. Also, the patient developed panhypopituitarism during this time. After an all around assessment we suspected hypopthsitis and transsphenoidal pituitary biopsy was performed. Non-necrotizing granulomatous hypophysitis was detected in histological examination. Causes of secondary granulomatous hypophysitis such as sarcoidosis, TBC, Langerhans cell histiocytosis, Wegener and syphilis were excluded and patient was diagnosed as primary granulomatous hypophysitis. After reviewing the available literature, hypophysectomy and high dose corticosteroid treatment seems to be effective for decreasing the symptoms but neither of them had an effect on curing panhypopituitarism when developed before these treatments. In our case; symptoms were regressed and panhypopituitarism was already developed. Therefore, we did not find it necessary to perform additional surgical intervention or giving high dose corticosteroid therapy. Recently initiated replacement therapies were continued, and the patient was subjected to followup.

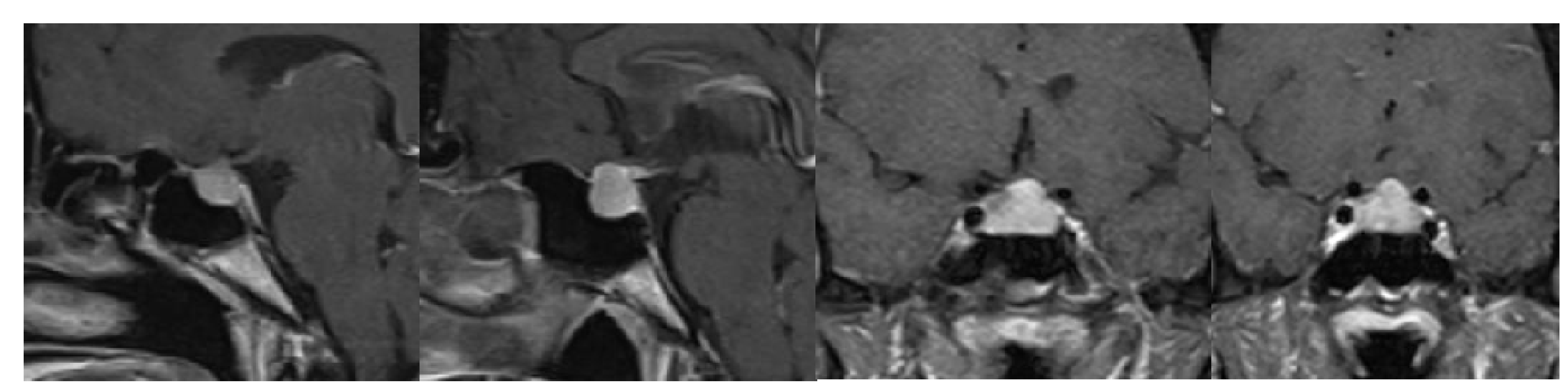

Figure 1. Initial pituitary MRI

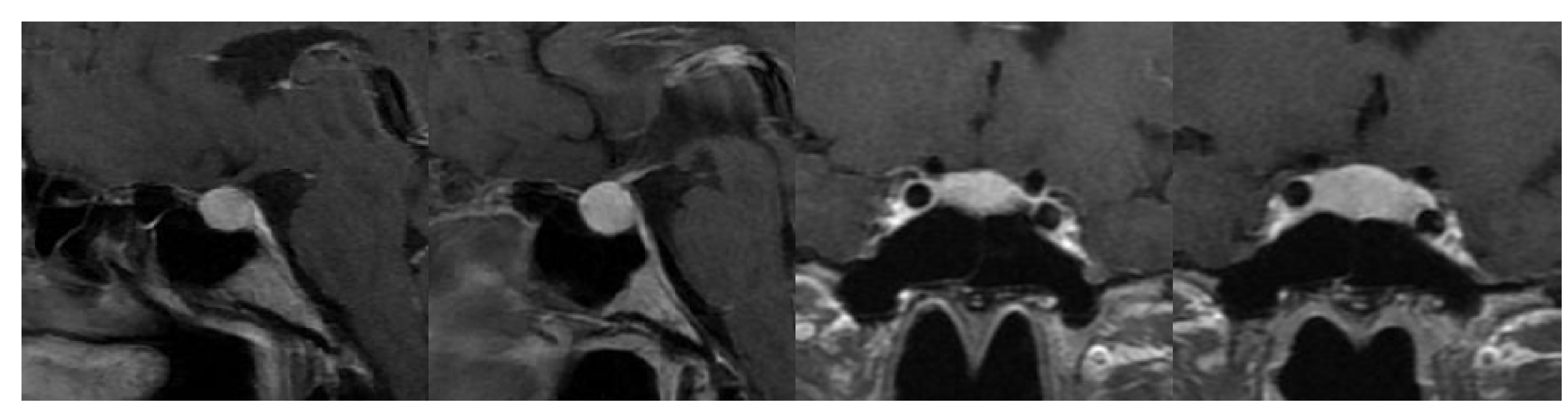

Figure 2. Follow-up pituitary MRI

\section{Discussion}

Even though the radiological findings can provide information for adenoma-hypophysitis differentiation, it is hardly possible to make differential diagnosis only by radiological assessment. Today, most of the cases are undergoing surgery and diagnosed post-operatively. However, there's a chance to diagnose hypophysitis preoperatively and treat it with steroids.

This case indicates that a careful review of radiological findings and clinical clues can lead endocrinologists to consider the possibility of hypophysitis with reasonable certainty and treat with steroids. This way, hypophysitis can be diagnosed preoperatively or with pituitary biopsy if necessary. This methods may be able to eliminate surgery and thus avoid short, long-term surgical complications.

This case report also reveals that more research needs to be done regarding to differential diagnosis of hypophsitis and its treatment with high-dose or normal dose steroid therapy. The diagnosis, in particular, needs to be done more effectively without the intervention of surgery. 